\title{
Conceptos Biomecánicos de las Suturas Tendinosas
}

\section{Biomechanical Concepts of Tendon Sutures}

\author{
Jaime Alonso Pérez-Barquero ${ }^{1}$ Elena Fernández Sabaté ${ }^{2}$ Eduardo Sánchez-Alepuz ${ }^{3}$
}

1 Unión de Mutuas, Hospital 9 de Octubre, Valencia, España

2 Unión de Mutuas, Valencia, España

3 Unión de Mutuas, Hospital IMED, Valencia, España

Address for correspondence Jaime Alonso Pérez-Barquero, Unión de Mutuas, Hospital 9 de Octubre, Valencia

Rev Iberam Cir Mano 2018;46:143-149.

\section{Resumen \\ Palabras clave \\ - Biomecánica \\ - reparación tendón flexor \\ - injerto tendinoso \\ - transferencia tendinosa}

El objetivo de una sutura tendinosa es que sea lo suficientemente resistente para permitir una movilidad activa precoz. Para ello, los cirujanos deben comprender las características biomecánicas de las suturas tendinosas. El objetivo de este artículo es recoger los aspectos fundamentales de los estudios biomecánicos, así como los principales factores que influyen en la resistencia de una sutura, tanto en tenorrafias primarias como en el caso de injertos y transferencias. Estudios in vitro o in vivo, con tracción lineal o curvilínea, cíclicos o de tracción única, son algunos de los factores que hemos de tener en cuenta a la hora de analizar un estudio sobre suturas tendinosas, así como conocer los principales parámetros físicos que analizan. Así mismo el material, el diámetro, la configuración, el número de hebras, el tipo de agarre al tendón, la tensión de la sutura, el tipo de nudo y la configuración de la sutura epitendinosa son los principales factores que influyen en la resistencia de las suturas primaria. En el caso de los injertos y transferencias, el número de entrelazaos y el tipo de puntos utilizados, son los principales aspectos que influyen en la sutura tipo Pulvertaft y sus alternativas.

The goal of a tendon suture is to be strong enough to allow active early mobility. Surgeons must understand the biomechanical characteristics of tendon sutures. The aim of this article is to collect the fundamental aspects of biomechanical studies as well as the main factors that influence the strength of a tendon suture, both in primary tenorrhaphies and in tendon grafts and transfers. Studies in vitro or in vivo, with linear or curvilinear traction, with cyclic or single traction, are some of the factors that we have to take into account when analyzing a study of tendon suture, as well as knowing the main physical parameters. Likewise, the material, the diameter, the configuration, the number of strands, the type of grip to the tendon, the tension of the suture, the knot and the configuration of the epithendinous suture are the main factors that influence the resistance of the sutures. In the case of grafts and transfers, the number of weaves and the type of stitch are the main aspects that influence in the Pulvertaft weave suture and its alternatives.

\section{Introducción}

El objetivo principal de una sutura tendinosa es que sea lo suficientemente resistente para que permita una movilidad activa precoz, ya que supone una mejora en los resultados de las reparaciones al disminuir las adherencias y mejorar la cicatrización de los tendones. ${ }^{1-3}$ Una técnica de sutura ideal debe presentar la suficiente resistencia para prevenir su received

September 11, 2018

accepted

September 27, 2018

published online

November 1, 2018
DOI https://doi.org/

10.1055/s-0038-1675390. ISSN 1698-8396.
Copyright (C 2018 Thieme Revinter

Publicações Ltda, Rio de Janeiro, Brazil
License terms

() (1) $\odot \circledast$ 
rotura y la formación de un gap a la vez que presenta una mínima reacción y lesión en el tendón. ${ }^{2}$

El método de sutura más comúnmente utilizado consta de una sutura central o core y una sutura epitendinosa, 3,4 mientras que en el caso de la sutura de tendones diferentes para injertos o transferencias tendinosas no se hace dicha distinción.

Cada cirujano debe elegir su técnica de elección teniendo en cuenta las características biomecánicas que presenta dicha sutura, y para ello debemos entender dichos estudios. El presente texto pretende recoger los aspectos fundamentales de los estudios biomecánicos, así como los conceptos más destacados de las suturas desde un punto de vista únicamente biomecánico.

\section{Estudios Biomecánicos de las Suturas Tendinosas}

\section{Modelos Utilizados}

In vitro: este tipo de trabajos son los más frecuentes en la literatura. Se trata de estudios que analizan desde el punto de vista biomecánico las suturas en el momento cero de la reparación sin tener en cuenta la capacidad de cicatrización del tendón. Los más frecuentemente utilizados cuando se trata de estudios in vitro son los tendones humanos y porcinos, ${ }^{5-8}$ aunque también se han utilizado de perro, ${ }^{9}$ oveja, ${ }^{10}$ conejo $^{11} \mathrm{y}$ gallina. ${ }^{12}$ Es importante conocer que existen diferencias desde el punto de vista biomecánico. Así, la fuerza necesaria para desgarrar un punto bloqueado a nivel de la zona II de un flexor porcino y ovino es mayor que en el tendón humano, y eso se debe a la diferencia en la microestructura que presenta mayores ondulaciones haciéndolos más resistentes. ${ }^{13}$ Por tanto, no debemos comparar las conclusiones de dos estudios que utilicen tendones de especies diferentes ni extrapolar resultados absolutos (en cuanto resistencia de la sutura), de dichos estudios, a nuestra práctica clínica habitual. Lo ideal, cuando se trata de estudios in vitro, es utilizar tendones humanos, aunque su principal limitación es la disponibilidad.

In vivo: Son mucho más escasos por su dificultad y por las limitaciones éticas que presentan. Descartados los tendones humanos por razones obvias, los conejos ${ }^{11}$ y recientemente los pavos ${ }^{14}$ son los modelos más utilizados. Tiene la ventaja de que son capaces de medir las propiedades biomecánicas en las fases tempranas de la curación. Ese hecho cobra importancia ya que sabemos que la resistencia de los tendones disminuye los primeros días siendo mínima a los 5-7 días en el caso de reparaciones primarias y a las 2 semanas en el caso de los injertos o transposiciones. ${ }^{11}$ Los tendones de los conejos son extrasinoviales y simulan el ambiente de los injertos o transferencias tendinosas, pero son tendones más elásticos y gomosos que los humanos. ${ }^{15}$

\section{Test Biomecánicos}

Opciones de Máquinas y Tipos de Ensayos

La práctica totalidad de los estudios biomecánicos se llevan a cabo con máquinas de carga axial que realizan una tracción de ambos extremos tendinosos linealmente (-Fig. 1).

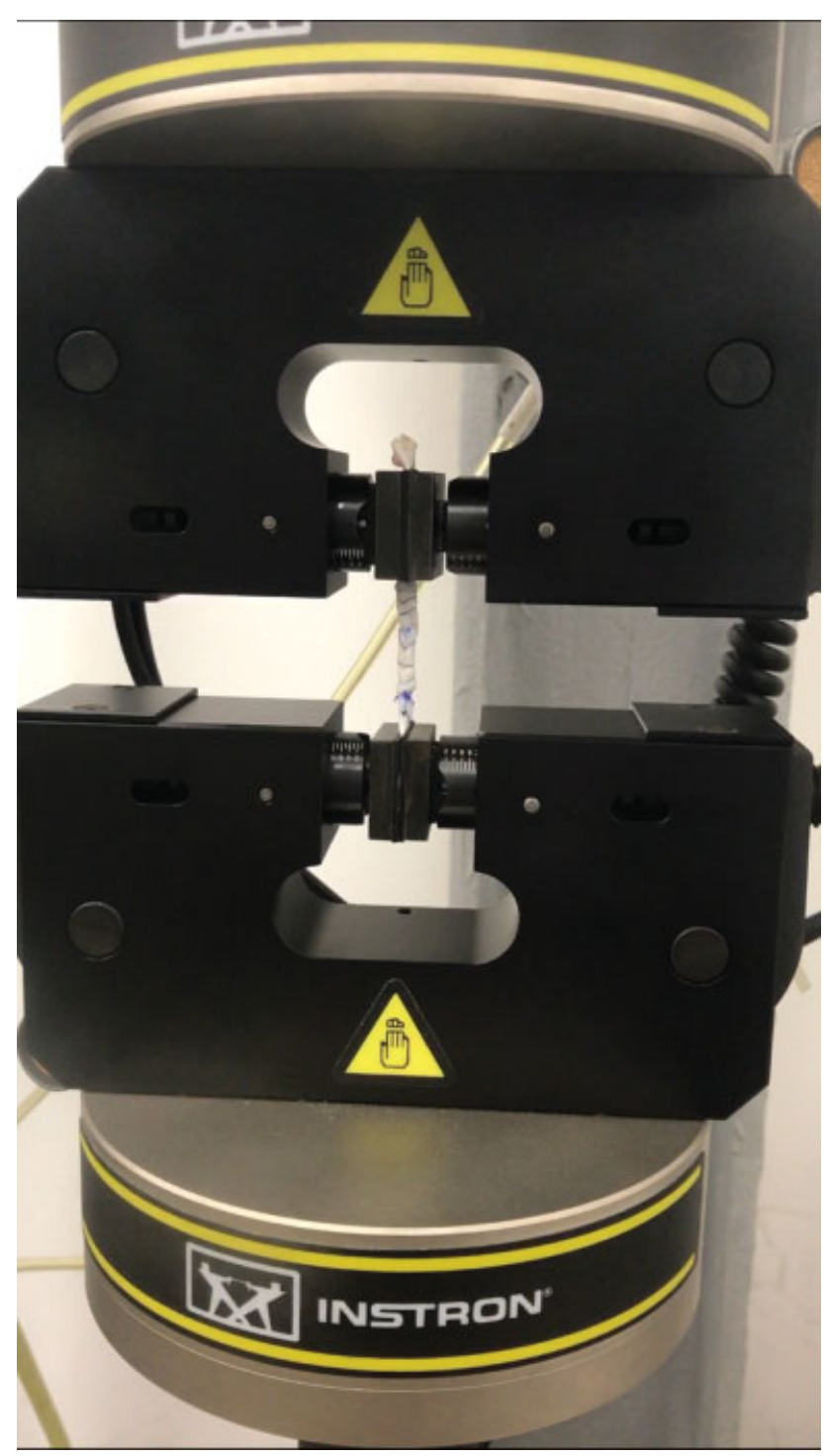

Fig. 1 Modelo de máquina de tracción axial.

Existen además contados artículos ${ }^{3,16}$ que utilizan un modelo de tracción curvilíneo para simular de una manera más real el movimiento del tendón, en el cual durante la flexión del dedo se produce una concentración de las fuerzas en el vértice de la curvatura. ${ }^{3}$

Además, los estudios pueden realizarse mediante una única tracción hasta alcanzar el punto máximo de rotura, o por el contrario realizar un ensayo cíclico en el cual se intenta analizar el comportamiento de la sutura ante movimientos repetidos. ${ }^{5,6}$

Datos más Importantes de los Estudios Biomecánicos De cada ensayo de tracción se obtiene una curva de cargadeslizamiento ( - Fig. 2) hasta obtener el fallo de la sutura y a partir de dicha curva se obtienen algunos datos necesarios:

- Fuerza máxima: representado por el punto más alto de la gráfica, siendo su unidad de medida el newton(N). Es el valor que más importancia adquiere en los estudios biomecánicos, probablemente por su fácil interpretación y por el hecho de que no permite muchas interpretaciones subjetivas. 


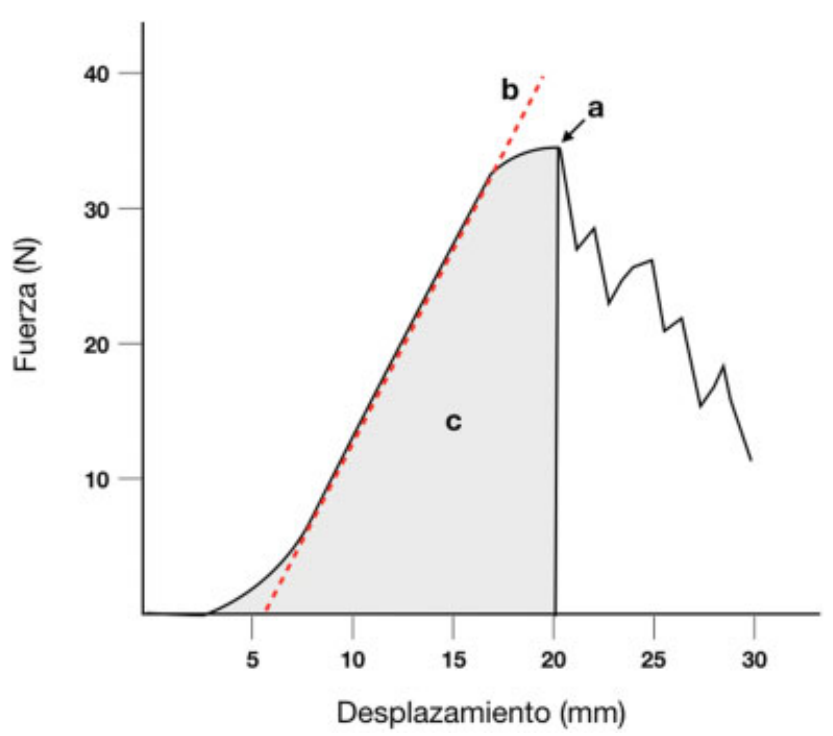

Fig. 2 Modelo de gráfica de fuerza-desplazamiento obtenida tras un ensayo de tracción. Fuerza máxima (a), rigidez (b) y energía absorbida (c) son los principales datos que se extraen de ella.

- Rigidez: se obtiene calculando la pendiente lineal trazada en la parte media de la curva y se representa en forma de razón entre la fuerza aplicada y el desplazamiento obtenido, en nuestro caso $\mathrm{N} / \mathrm{mm}^{2}$. Su uso e interpretación en los estudios biomecánicos de suturas tendinosas es discutido, ya que se duda que una mayor rigidez en un tendón reparado se traduzca en un beneficio clínico.

- Energía absorbida: se obtiene calculando el área bajo la curva de la gráfica de carga-desplazamiento y no está claro la transcendencia clínica que presenta dicha variable.

- Resistencia al gap: el objetivo de los estudios que tratan de medir ese parámetro consiste en averiguar la fuerza necesaria para que aparezca un gap. La mayoría de los artículos, utilizan los $2 \mathrm{~mm}$ como medida estándar, aunque existen los que sitúan los $3 \mathrm{~mm}$ como límite para una correcta cicatrización. ${ }^{2,8}$ La aparición del gap se traduce clínicamente en una peor cicatrización, mayor formación de adherencias y atrapamientos a nivel de las poleas. ${ }^{3}$ La mayoría de estudios que tratan de analizar el gap lo hacen midiéndolo tras un ciclo de cargas, ya que si se utiliza una tracción lineal hasta el fallo no puede medirse. Al margen del método utilizado, la resistencia al gap, junto a la fuerza máxima resultan los datos de mayor relevancia a la hora de analizar un estudio biomecánico de suturas tendinosas. Únicamente destacar que en el caso de suturas que presenten una aposición de los extremos, como en el caso de los injertos o transferencias, el cálculo de la resistencia al gap deja de tener sentido.

- La fuerza de fatiga: utilizado en pocos artículos ha sido definida como el producto de la fuerza aplicada por el número de ciclos antes de la rotura. ${ }^{8}$ Presenta el mismo significado clínico incierto que la energía absorbida, pero en este caso más propios para estudios cíclicos.

\section{Biomecánica de las Reparaciones Primarias}

\section{Sutura del Core}

La sutura del core influye principalmente en la fuerza máxima, rigidez y energía absorbida, y parcialmente en la resistencia al gap. Los principales factores que influyen en la sutura del core son:

- Material de sutura: facilidad de uso, mínima reacción de los tejidos y mayor fuerza son las características deseadas de un material de sutura. Existen multitud de materiales disponibles, y actualmente la preferencia del cirujano suele venir dada por su experiencia particular más que por una evidencia científica. ${ }^{2}$ Diferentes estudios han concluido que la sutura de Ethibond (Ethicon, Someville, NJ, USA), presenta una mayor resistencia cuando se le compara con Ethilon (Ethicon, Someville, NJ, USA), Prolene (Ethicon, Someville, NJ, USA) y Supramid (S. Jackson, Alexandria, VA, USA). ${ }^{17,18}$ En los últimos años la utilización del FiberWire (Arthrex, Naples, FL) para las suturas tendinosas ha aumentado. ${ }^{2}$ Dicha sutura ha demostrado resistencias superiores a la del Ethibond, Nylon y Prolene, de hecho, Brockardt y cols., demostraron que una sutura de Kessler de dos hebras con FiberWire (Arthrex, Naples, FL) equivalía a una sutura de 4 hebras cruzadas con Supramid. ${ }^{17}$ Se han llegado a publicar fuerzas de hasta $124 \mathrm{~N}$ cuando se ha utilizado el loop de FiberWire (Arthrex, Naples, FL) en la sutura LimTsai modificada de 6 hebras. ${ }^{19}$ Pero el FiberWire (Arthrex, Naples, FL) no está exento de problemas, ya que presenta un diámetro mayor que la misma categoría que otras suturas lo que significa que si medimos la resistencia de la sutura en proporción a su área transversal, esta sólo sea un $10 \%$ más fuerte que el Ticron (Syneture, Norwalk, CT, USA), ${ }^{20}$ además su dificultad para obtener un anudado seguro ha provocado la aparición de estudios que analizan la configuración del nudo y su resistencia en ese tipo de sutura. $^{21-23}$ Tal es así que cuando se comparan Ethibond, Ethilon, Surgilon y PDS II y se utiliza un anudado quirúrgico habitual, Surgilon (multifilamento de Nylon), presenta una mayor resistencia a la rotura, en cambio, cuando la sutura se realiza con 4 bucles adicionales al anudado habitual, FiberWire (Arthrex, Naples, FL) presenta la mayor resistencia. ${ }^{24}$

Aunque algunos autores han demostrado que las suturas absorbibles (Maxon, PDS...) presentan resistencias que permiten una movilización precoz son cada vez menos utilizadas para las suturas tendinosas por su pérdida de fuerza en el tiempo y su elevada elasticidad. ${ }^{2}$

- Diámetro de la sutura: los calibres más utilizados en suturas tendinosas del core son el 3-0 y el 4-0, aunque se han utilizado también suturas de 2-0 (abandonado por su excesivo aumento del volumen a nivel de la sutura) y 50 (poco utilizado por su insuficiente resistencia). ${ }^{2}$ Test biomecánicos han demostrado que la sutura 3-0 en comparación con la 4-0 presenta un aumento de la fuerza de alrededor del 50\%,25,26 aunque utilizados en una sutura de 4 hebras cruzadas y bloqueada la sutura 3-0 
no ha demostrado ser superior en cuanto a fuerza máxima y resistencia al gap que la de $4-0 .{ }^{27}$ Como veremos más adelante, el número de hebras que atraviesan el core es otro de los factores que influyen en la resistencia de una sutura y una de las limitaciones del uso de suturas 3-0 es su dificultad a la hora de realizar configuraciones de 8 hebras con el fin de aumentar la resistencia, ya que presentan un área transversal desproporcionada en comparación con la del tendón para llevar a cabo ese tipo configuraciones. Siguiendo esa línea, Osei y cols., demostraron que la utilización de una sutura tipo Ethibond de 4-0 y 8 hebras resulta un 43\% más resistente que la misma sutura de $3-0$ y 4 hebras. $^{26}$ Aunque no puede tomarse como una afirmación estricta, por regla general hemos de pensar en suturas de 3-0 para configuraciones de 4 hebras y suturas de 4-0 para configuraciones de 6 y 8 hebras ${ }^{28}$

- La configuración de la sutura: existen trabajos que comparan dos suturas diferentes que utilizan el mismo material, diámetro, número de hebras. Kang y cols., han postulado que la mayor resistencia al gap en estudios cíclicos de la sutura tipo Tang vs la Lim-Tsai modificada se debe a que la primera utiliza suturas multiplanares y tres hebras dobles independientes en comparación a la sutura uniplanar y con una hebra doble continua que utiliza la Lim-Tsai modificada. Ninguno de esos factores -suturas multiplanares y uso de una hebra contínua o varias hebras- ha sido estudiado de manera independiente y por tanto es difícil afirmar que se trate de factores que influyen positivamente en la resistencia de la sutura. Desde un punto de vista estricto únicamente es cierto que una configuración determinada es superior a la otra, el resto de afirmaciones todavía no han sido probadas científicamente. De igual modo, Kozono y cols ${ }^{8}$ encuentran en la sutura asimétrica de Pennington, una mayor resistencia a la fatiga cuando se le compara con las suturas triple loop (tres suturas dobles tipo Tsuge) y con la sutura Yoshizu \#1, utilizando en los tres casos el mismo número de hebras y el mismo material (Monofilamento Nylon 4-0).

- Número de hebras: Es una creencia ampliamente aceptada que el aumento del número de hebras que atraviesan el core supone un aumento proporcional de la fuerza máxima de la sutura así como de la resistencia al gap. ${ }^{2,29}$ En un estudio in vivo, Winters y cols., demostraron que a las 3 semanas de la reparación la fuerza máxima de una sutura de 8 hebras es un $49 \%$ mayor ascendiendo al $117 \%$ a las 6 semanas cuando se le compara con configuraciones de 4 hebras. $^{30}$ Recientemente, algún estudio ha puesto en entredicho esa afirmación. ${ }^{31}$ Dichos autores han acuñado un nuevo término denominado "eficiencia constructiva" de una sutura, afirmando que la resistencia final de la sutura no es proporcional al número de hebras utilizadas multiplicadas por la resistencia de una única hebra de dicho material, haciendo hincapié en la disposición de dicha sutura. ${ }^{32}$ Aún así los autores del presente artículo pensamos que no se ha de confundir y mezclar ambos conceptos. Resulta lógico pensar que a mayor número de hebras mayor resistencia de la sutura, aunque dicho aumento pudiera no ser siempre directamente proporcional, y por otro lado resulta obvio pensar que la disposición de la sutura también influye en la resistencia de la sutura, son dos conceptos compatibles no excluyentes. La utilización de suturas con doble loop para aumentar la resistencia de las suturas tendinosas es un hecho controvertido actualmente. Así, Calfee y cols., afirman que las propiedades mecánicas de una sutura -Kesler modificado- utilizando sutura simple es significativamente mayor que las que utilizan suturas tipo loop. ${ }^{33}$ Aunque no se trata de un aspecto biomécanico, la utilización de las suturas tipo loop tienen la ventaja de permitirnos aumentar el número de hebras con la mitad de pasos a través de los extremos del tendón, lo que da lugar a suturas más fáciles de realizar, y este es un hecho que al margen de las discusiones sobre las suturas con loop o simples debe ser tenido en cuenta a la hora de elegir la sutura más adecuada. Pero es precisamente el mayor número de pasos a través del tendón, obteniendo así más interacciones entre el tendón y la sutura lo que postulan los autores, es el motivo de que las suturas simples tengan mayores propiedades mecánicas que las suturas tipo loop. ${ }^{33}$ Hasta hace unos años, la amplia mayoría de cirujanos, utilizaban técnicas de 4 hebras pues resulta un buen equilibrio entre la fuerza máxima y la facilidad de realización de la sutura, ${ }^{34}$ sin embargo, la aparición de nuevas configuraciones y suturas tipo loop ha hecho que cada vez más se utilicen configuraciones de 6 u 8 hebras. ${ }^{5-7}$

- El tipo de agarre al tendón: aunque existen numerosos estudios que defienden que los agarres bloqueados aumentan la resistencia, recientemente Lim y cols han postulado que el aumento de la resistencia de la sutura asimétrica Lim-Tsai respecto a la Lim-Tsai modificada podría deberse a la utilización de puntos de anclaje no bloqueados al tendón, lo que originaría una transmisión más suave de las cargas a través de las hebras. ${ }^{6}$

- La tensión de la sutura: se trata de uno de los factores menos estudiados por su dificultad en la aplicación clínica, ya que hasta la fecha no resulta posible cuantificar el acortamiento tendinoso a costa de una determinada tensión a nivel de la sutura del core. A pesar de ello, se recomienda un pretensado de las suturas en cada paso, así como antes del anudado con el objetivo de equilibrar las cargas que soportan cada una de las hebras. ${ }^{2}$ Dicha tensión influye positivamente en la fuerza de gap y en la rigidez pero parece que tiene poca influencia en la fuerza máxima. ${ }^{3}$ Estudios biomecánicos han demostrado que un acortamiento del $10 \%$ del tendón supone un aumento significativo de la fuerza de gap sin que ello suponga un aumento del volumen a nivel de la sutura. ${ }^{35}$

- Tipo de nudo y su localización: El tipo de nudo a realizar en cada sutura es un aspecto que ha cobrado importancia con la aparición de suturas sintéticas como el Fiberwire (Arthrex, Naples, FL, USA). Partiendo de estudios previos que afirmaban la necesidad de utilizar más vueltas en los nudos con ese material para evitar el fracaso de la sutura ${ }^{23}$ con el consiguiente aumento de volumen de la sutura, 
Jiang y $\operatorname{cols}^{36}$ platearon un estudio acerca del tipo de nudo más resistente en suturas tipo FiberWire (Arthrex, Naples, FL, USA), concluyendo que el nudo anti-slip presenta la mayor resistencia pero a costa de un mayor volumen del mismo. Aún así, los autores aconsejan el nudo $1=1=1=1=1$ por presentar la mejor relación fuerza/volumen y por ser un nudo que presenta suficiente fuerza para realizar una rehabilitación con movilidad activa precoz. En cuanto a la localización del nudo Chang y cols concluyen en uno de sus últimos trabajos que, modificando la localización del nudo en la sutura Lim-Tsai modificada y colocándolo extratendinosamente, se aumenta la fuerza máxima y la resistencia al gap, al obtener una mejor distribución de las cargas. ${ }^{7}$ Aunque extrapolar dicho concepto a todas las suturas puede resultar una equivocación, trabajos como este y el hecho de que la colocación del nudo intratendinosamente aumenta el material en la zona de cicatrización, han de tenerse en cuenta a la hora de elegir la sutura más adecuada. El término extra o intratendinosamente utilizado habitualmente se refiere a la colocación de un nudo entre ambos extremos tendinosos lo que origina una mayor interposición de material en el lugar de la cicatrización o a la colocación del mismo sobre la superficie el tendón a cierta distancia de los extremos seccionados, pero dichos términos deben ser actualizados, pues existen suturas ${ }^{37}$ en las que el nudo se encuentra a distancia de la zona de reparación pero intratendinosamente, lo que evita los problemas de deslizamiento que ocasiona la localización del nudo sobre la superficie del mismo.

\section{Sutura Epitendinosa}

A la sutura epitendinosa se le otorga una capacidad de mejora de la biomecánica de las suturas, especialmente a expensas de mejorar la resistencia al gap, no siendo un factor que influya significativamente en la fuerza máxima, ${ }^{38}$ aunque el porcentaje de aumento de la fuerza máxima y de la resistencia al gap es diferente según el tipo de sutura del core. ${ }^{39}$ De hecho, estudios in vivo y ex vivo han demostrado que la configuración de la sutura epitendinosa -recomendando un agarre de $2 \mathrm{~mm}$-, junto al diámetro de la sutura y número de hebras del core suponen factores principales en la mejora de la resistencia de una sutura, mientras que el tamaño de la sutura epitendinosa y el tipo de agarre de la sutura del core suponen factores secundarios. ${ }^{40,41}$

La sutura epitendinosa cruzada ha demostrado soportar cargas un $245 \%$ mayores que las soportadas por la sutura epitendinosa simple continua. ${ }^{42}$ Aún así la sutura epitendinosa simple continua es ampliamente utilizada por su fácil realización. ${ }^{8}$

Aunque como hemos visto la sutura epitendinosa tiene la capacidad de aumentar la resistencia de la sutura, su principal función se ha relacionado con la mejora del volumen en la zona de unión de ambos extremos reparados con el fin de mejorar el deslizamiento, evitando la formación de adherencias.

\section{Biomecánica de las Reconstrucciones Tendinosas}

\section{Técnica Pulvertaft y los Factores que Influyen en su Resistencia}

La unión de dos tendones mediante el entrelazado de Pulvertaft (PT) descrita hace más de 60 años, ${ }^{43}$ continúa siendo el método gold estándar para injertos y transferencias tendinosas.

En cuanto al número de entrelazados que deben realizarse existe controversia. Existen estudios que han demostrado un aumento lineal de la fuerza máxima directamente proporcional al número de entrelazados desde uno a cuatro, ${ }^{44}$ mientras que otros autores no encuentran diferencias entre 2 y 3 entrelazados siendo la diferencia estadísticamente significativa cuando se utilizaron 4 entrelazados. ${ }^{45}$

Gabuzda y $\operatorname{cols}^{44}$ demostraron que la utilización de puntos cruzados presentaban mayores valores de fuerza máxima que los obtenidos con puntos horizontales, hasta el punto que 3 entrelazados con puntos cruzados presentaban una resistencia comparable a 5 entrelazados con puntos horizontales. Tanaka y cols ${ }^{46}$ proponen puntos a nivel de las esquinas de las uniones tendinosas en cada lazada pues resultan tan resistentes como los puntos cruzados, pero al ser más superficiales presuponen que permite una mejor cicatrización de los tendones al afectar menos la vacularización.

Se ha de tener en cuenta que el tamaño de los tendones que se utilizan como donantes influyen en la resistencia final de la sutura, ya que la unión mediante PT de tendones flexores superficiales y profundos presentan fuerzas máximas que permiten una movilización precoz, siendo ellas muy inferiores cuando el donante es un tendón palmar largo y la mitad cuando el donante es un plantar delgado. ${ }^{47}$

\section{Otras Técnicas Propuestas en la Literatura}

- Side to Side: descrito por Brown y cols., utiliza solo un entrelazado y posteriormente ambos extremos se solapan uno a otro y se suturan con puntos cruzados en ambos lados. ${ }^{48}$ Dicha técnica presenta valores superiores a los de PT en cuanto a fuerza máxima se refiere, con un volumen a nivel de la unión tendinosa similar en estudio sobre tendones extensores humanos. ${ }^{49}$

- Técnica Lasso: descrita por Bidic y cols., presenta una fuerza máxima ligeramente inferior a la PT siendo no significativa esa diferencia. ${ }^{50}$ Los autores defienden su uso, por su facilidad y rapidez y porque necesita menos longitud de tendón, aunque la unión resultante es más voluminosa que la PT.

- Técnica Espiral: presenta fuerzas máximas similares al PT, presentando un mayor volumen a nivel de la sutura y una mayor necesidad de longitud tanto del tendón receptor como del donante. ${ }^{51}$

- Técnica Loop: De Smet y cols ${ }^{52}$ presentaron los resultados de un estudio biomecánico donde la unión tipo loop presentaba una mayor fuerza máxima respecto al PT, pero consideraban una limitación la poca superfice de 
contacto que existía entre ambos tendones lo que podría provocar una peor cicatrización. Pero dicha suposición quedó desmentida con el estudio in vivo donde la unión tipo loop demostró presentar una mayor fuerza máxima y energía absorbida cuando se comparó con una técnica de entrelazado en ambos extremos -no era una técnica PT convencional- a las tres semanas de la sutura, no existiendo diferencias a las 4 semanas. Cabe destacar que ese trabajo demostró que la disminución de la fuerza máxima presenta su pico más bajo a las 2 semanas de la cirugía ${ }^{11}$

- Técnica Step-Cut: descrito por Becker y cols., donde ambos extremos son cortados oblicuamente para después solaparlos siendo su objetivo principal no aumentar en exceso el volumen a nivel de la unión tendinosa. ${ }^{53}$ Estudios realizados en modelos caninos no presentan diferencias biomecánicas comparadas con el PT pero sî un menor volumen en la unión tendinosa ${ }^{9}$

- Cow-Hitch: Dicha téncica que utiliza una lazada de un tendón sobre el otro, a pesar de mostrar una mayor fuerza máxima que el PT presenta dos limitaciones importantes, ya que uno de los extremos presenta dos cabos tendinosos y además debe hacerse con uno de los dos tendones libres, por lo que no serviría para injertos o transferencias donde en primer lugar se realiza la fijación distal. ${ }^{10}$

- Wrap-around: técnica en la que uno de los extremos "envuelve" al otro y que ha demostrado ser igual o superior a la PT con un menor volumen en la unión tendinosa en modelos porcinos. ${ }^{45}$

\section{Conclusiones}

El cirujano de mano debe conocer los factores que influyen en la resistencia de las suturas tendinosas tanto en tenorrafias primarias como en injertos y transferencias a la hora de tomar una decisión para elegir el tipo de sutura ideal para cada caso de su práctica clínica habitual. Comprender los principales aspectos de los estudios biomecánicos ayudará en esa difícil labor de interpretación de los resultados de dichos estudios.

\section{Conflict of Interest}

No hay conflcitos de interés.

\section{Bibliografía}

1 Tang JB, Amadio PC, Boyer MI, et al. Current practice of primary flexor tendon repair: a global view. Hand Clin 2013;29(02):179-189

$2 \mathrm{Wu}$ YF, Tang JB. Recent developments in flexor tendon repair techniques and factors influencing strength of the tendon repair. J Hand Surg Eur Vol 2014;39(01):6-19

3 Bo Tang J, Guo Xie R. Biomechanics of Core and Peripheral Tendon Repairs. En: Elsevier Inc (Ed). Tendon Surgery of the Hand. 2012: 35-48

4 Kubota H, Aoki M, Pruitt DL, Manske PR. Mechanical properties of various circumferential tendon suture techniques. J Hand Surg [Br] 1996;21(04):474-480

5 Kang GH-Y, Wong YR, Lim RQ-R, Loke AMK, Tay SC. Cyclic Testing of the 6-Strand Tang and Modified Lim-Tsai Flexor Tendon Repair Techniques. J Hand Surg Am 2018;43(03):285.e1-285.e6
6 Lim RQR, Wong YR, Loke AMK, Tay SC. A cyclic testing comparison of two flexor tendon repairs: asymmetric and modified Lim-Tsai techniques. J Hand Surg Eur Vol 2018;43(05):494-498

7 Chang MK, Wong YR, Tay SC. Biomechanical comparison of modified Lim/Tsai tendon repairs with intra- and extra-tendinous knots. J Hand Surg Eur Vol 2018;24:1753193418769804

8 Kozono N, Okada T, Takeuchi N, Shimoto T, Higaki H, Nakashima Y. A Biomechanical Comparison Between Asymmetric Pennington Technique and Conventional Core Suture Techniques: 6-Strand Flexor Tendon Repair. J Hand Surg Am 2018;43(01):79.e1-79.e8

9 Hashimoto T, Thoreson AR, An KN, Amadio PC, Zhao C. Comparison of step-cut and Pulvertaft attachment for flexor tendon graft: a biomechanics evaluation in an in vitro canine model. J Hand Surg Eur Vol 2012;37(09):848-854

10 Vincken NLA, Lauwers TMAS, van der Hulst RRWJ. Biomechanical and Dimensional Measurements of the Pulvertaft Weave Versus the Cow-Hitch Technique. Hand (N Y) 2017;12(01):78-84

11 Jeon SH, Chung MS, Baek GH, Lee YH, Kim SH, Gong HS. Comparison of loop-tendon versus end-weave methods for tendon transfer or grafting in rabbits. J Hand Surg Am 2009;34(06):1074-1079

12 Kim SH, Chung MS, Baek GH, Lee YH, Lee S, Gong HS. A looptendon suture for tendon transfer or graft surgery. J Hand Surg Am 2007;32(03):367-372

13 Peltz TS, Hoffman SW, Scougall PJ, et al. Animal Models for Tendon Repair Experiments: A Comparison of Pig, Sheep and Human Deep Flexor Tendons in Zone II. J Hand Surg Asian Pac Vol 2017;22(03): 329-336

14 Kadar A, Liu H, Vrieze AM, et al. Establishment of an in vivo turkey model for the study of flexor tendon repair. J Orthop Res 2018;36 (09):2497-2505

15 An YH, Freidman RJ. Animal Models in Orthopaedic Research. CRC Press; 1998:1

16 Miller L, Mass DP. A comparison of four repair techniques for Camper's chiasma flexor digitorum superficialis lacerations: tested in an in vitro model.J Hand Surg Am 2000;25(06):1122-1126

17 Brockardt CJ, Sullivan LG, Watkins BE, Wongworawat MD. Evaluation of simple and looped suture and new material for flexor tendon repair. J Hand Surg Eur Vol 2009;34(03):329-332

18 Mishra V, Kuiper JH, Kelly CP. Influence of core suture material and peripheral repair technique on the strength of Kessler flexor tendon repair. J Hand Surg [Br] 2003;28(04):357-362

19 Gan AWT, Neo PY, He M, Yam AKT, Chong AKS, Tay SC. A biomechanical comparison of 3 loop suture materials in a 6strand flexor tendon repair technique. J Hand Surg Am 2012;37 (09):1830-1834

20 Scherman P, Haddad R, Scougall P, Walsh WR. Cross-sectional area and strength differences of fiberwire, prolene, and ticron sutures. J Hand Surg Am 2010;35(05):780-784

21 Lee SK, Goldstein RY, Zingman A, Terranova C, Nasser P, Hausman MR. The effects of core suture purchase on the biomechanical characteristics of a multistrand locking flexor tendon repair: a cadaveric study. J Hand Surg Am 2010;35(07):1165-1171

22 Moriya T, Larson MC, Zhao C, An KN, Amadio PC. The effect of core suture flexor tendon repair techniques on gliding resistance during static cycle motion and load to failure: a human cadaver study. J Hand Surg Eur Vol 2012;37(04):316-322

23 Waitayawinyu T, Martineau PA, Luria S, Hanel DP, Trumble TE. Comparative biomechanic study of flexor tendon repair using FiberWire. J Hand Surg Am 2008;33(05):701-708

24 Komatsu F, Mori R, Uchio Y. Optimum surgical suture material and methods to obtain high tensile strength at knots: problems of conventional knots and the reinforcement effect of adhesive agent. J Orthop Sci 2006;11(01):70-74

25 Taras JS, Raphael JS, Marczyk SC, Bauerle WB. Evaluation of suture caliber in flexor tendon repair. J Hand Surg Am 2001;26(06): 1100-1104

26 Osei DA, Stepan JG, Calfee RP, et al. The effect of suture caliber and number of core suture strands on zone II flexor tendon 
repair: a study in human cadavers. J Hand Surg Am 2014;39(02): 262-268

27 Alavanja G, Dailey E, Mass DP. Repair of zone II flexor digitorum profundus lacerations using varying suture sizes: a comparative biomechanical study. J Hand Surg Am 2005;30(03):448-454

28 Barrie KA, Tomak SL, Cholewicki J, Merrell GA, Wolfe SW. Effect of suture locking and suture caliber on fatigue strength of flexor tendon repairs. J Hand Surg Am 2001;26(02):340-346

29 Thurman RT, Trumble TE, Hanel DP, Tencer AF, Kiser PK. Two-, four-, and six-strand zone II flexor tendon repairs: an in situ biomechanical comparison using a cadaver model. J Hand Surg Am 1998;23(02):261-265

30 Winters SC, Gelberman RH, Woo SL, Chan SS, Grewal R, Seiler JG III. The effects of multiple-strand suture methods on the strength and excursion of repaired intrasynovial flexor tendons: a biomechanical study in dogs. J Hand Surg Am 1998;23(01): 97-104

31 Wong YR, Lee CS, Loke AMK, Liu X, Suzana MJ I, Tay SC. Comparison of Flexor Tendon Repair Between 6-Strand Lim-Tsai With 4-Strand Cruciate and Becker Technique. J Hand Surg Am 2015;40(09):1806-1811

32 Wong YR, Loke AMK, Tay SC. The Effect of Suture Materials on the Biomechanical Performance of Different Flexor Tendon Repairs and the Concept of Construct Efficiency. J Hand Surg Asian Pac Vol 2018;23(02):243-247

33 Calfee RP, Boone S, Stepan JG, Osei DA, Thomopoulos S, Boyer MI. Looped versus single-stranded flexor tendon repairs: a cadaveric mechanical study. J Hand Surg Am 2015;40(05): 958-62.e1

34 Tang JB. Outcomes and evaluation of flexor tendon repair. Hand Clin 2013;29(02):251-259

$35 \mathrm{Wu}$ YF, Tang JB. Effects of tension across the tendon repair site on tendon gap and ultimate strength. J Hand Surg Am 2012;37(05): 906-912

36 Jiang J, Mat Jais IS, Yam AKT, McGrouther DA, Tay SC. A Biomechanical Comparison of Different Knots Tied on Fibrewire Suture. J Hand Surg Asian Pac Vol 2017;22(01):65-69

37 Corella F, Renner C, del Cerro M, Ocampos M. Técnica de sutura tendinosa «un paso, 4-hilos Kessler-Tsuge». Revista Iberoamericana Cirugía Mano 2015;43(02):122-127

38 Sull A, Inceoglu S, Wongworawat MD. Does Barbed Suture Repair Negate the Benefit of Peripheral Repair in Porcine Flexor Tendon? Hand (N Y) 2016;11(04):479-483

39 de Wit T, Walbeehm ET, Hovius SER, McGrouther DA. The mechanical interaction between three geometric types of nylon core suture and a running epitenon suture in repair of porcine flexor tendons. J Hand Surg Eur Vol 2013;38(07):788-794

40 Nelson GN, Potter R, Ntouvali E, et al. Intrasynovial flexor tendon repair: a biomechanical study of variations in suture application in human cadavera. J Orthop Res 2012;30(10):1652-1659

41 Fufa DT, Osei DA, Calfee RP, Silva MJ, Thomopoulos S, Gelberman $\mathrm{RH}$. The effect of core and epitendinous suture modifications on repair of intrasynovial flexor tendons in an in vivo canine model. J Hand Surg Am 2012;37(12):2526-2531

42 Kim PT, Aoki M, Tokita F, Ishii S. Tensile strength of cross-stitch epitenon suture. J Hand Surg [Br] 1996;21(06):821-823

43 Pulvertaft RG. Tendon grafts for flexor tendon injuries in the fingers and thumb; a study of technique and results. J Bone Joint Surg Br 1956;38-B(01):175-194

44 Gabuzda GM, Lovallo JL, Nowak MD. Tensile strength of the endweave flexor tendon repair. An in vitro biomechanical study. J Hand Surg [Br] 1994;19(03):397-400

45 Fuchs SP, Walbeehm ET, Hovius SER. Biomechanical evaluation of the Pulvertaft versus the 'wrap around' tendon suture technique. J Hand Surg Eur Vol 2011;36(06):461-466

46 Tanaka T, Zhao C, Ettema AM, Zobitz ME, An K-N, Amadio PC. Tensile strength of a new suture for fixation of tendon grafts when using a weave technique. J Hand Surg Am 2006;31(06):982-986

47 Mazurek T, Strankowski M, Ceynowa M, Rocławski M. Tensile strength of a weave tendon suture using tendons of different sizes. Clin Biomech (Bristol, Avon) 2011;26(04):415-418

48 Brown SHM, Hentzen ER, Kwan A, Ward SR, Fridén J, Lieber RL. Mechanical strength of the side-to-side versus Pulvertaft weave tendon repair. J Hand Surg Am 2010;35(04):540-545

49 Rivlin M, Eberlin KR, Kachooei AR, et al. Side-to-Side Versus Pulvertaft Extensor Tenorrhaphy-A Biomechanical Study. J Hand Surg Am 2016;41(11):e393-e397

50 Bidic SM, Varshney A, Ruff MD, Orenstein HH. Biomechanical comparison of lasso, Pulvertaft weave, and side-by-side tendon repairs. Plast Reconstr Surg 2009;124(02):567-571

51 Kulikov YI, Dodd S, Gheduzzi S, Miles AW, Giddins GE. An in vitro biomechanical study comparing the spiral linking technique against the pulvertaft weave for tendon repair. J Hand Surg Eur Vol 2007;32(04):377-381

52 De Smet L, Schollen W, Degreef I. In vitro biomechanical study to compare the double-loop technique with the Pulvertaft weave for tendon anastomosis. Scand J Plast Reconstr Surg Hand Surg 2008; 42(06):305-307

53 Becker $\mathrm{H}$, Davidoff $\mathrm{M}$. Eliminating the gap in flexor tendon surgery. A new method of suture. Hand 1977;9(03):306-311 